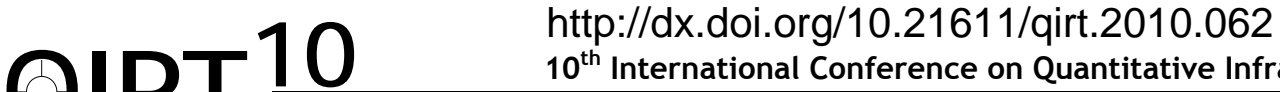 \\ $10^{\text {th }}$ International Conference on Quantitative InfraRed Thermography \\ July 27-30, 2010, Québec (Canada)
}

\section{Decision classification criteria for ADT in burn diagnostics based on in-vivo animal experiments}

by M. Kaczmarek*

*Biomedical Engineering Dept., Gdansk University of Technology, Gdansk, Poland, mariusz@biomed.eti.pg.gda.pl

\begin{abstract}
In this paper the decision classification criteria for burn wound diagnostic are described. Based on Active Dynamic Thermography (ADT) results from in-vivo animal experiments classification into two groups (wounds that will heal within 3 weeks and wounds that will not heal within 3 weeks) and on three classes $\left(I, I I^{\circ} \mathrm{A}\right.$ degree; $I I^{\circ} \mathrm{B}, \mathrm{IIl}$ degree) are discussed. This diagnostic tool should improve the treatment of burn wounds in clinical practice, resulting in shorter and cheaper treatment. It should be underlined that medical applications of QIRT seem to be the most difficult among others as heat transfers in living tissues are far more complicated comparing to technical applications using non-destructive testing based on thermal IR imaging (NDT TI).
\end{abstract}

1.

Introduction

Surgeons treating burns are of the opinion that conservative treatment should be applied to burn wounds that heal within three weeks of their occurrence, while surgery ought to be used for wounds that will not heal during this period.

Clinical estimation of burn wound depth, visual classification into 3 classes $10,1 \mathrm{I}^{\circ} \mathrm{A} \& \mathrm{~B}, \mathrm{II \textrm {I } ^ { \circ }}$, is subject to numerous errors. A complementary, and perhaps objective, diagnostic method could therefore be of a great importance. Of the methods currently used in this field, the following are worth mentioning: thermography [1], Laser Doppler Imaging (LDI) [2], indocyanine green (ICG) fluorescence [3], histopathologic examination of burn wound biopsies [4]. We trust that application of IR-thermal imaging supported by NDT procedures, mainly ADT and Thermal Tomography (TT) should allow for quantitative evaluation of burn depth and in consequence for proper identification of regions requiring surgical intervention. To prove this some in vivo experiments have been performed and several new procedures worked out as discussed later.

2.

Materials and Methods

The term Active Dynamic IR Imaging or Active Dynamic Thermography (ADT) is used to mean that information derived from IR thermal imaging data results from active external excitation, which forces temperature transients and is evidenced by dynamic changes at the surface of the object being tested.

The plan of the research was approved by the Local Ethics Commission for Experimentation on Animals at the Medical University of Gdansk, Poland. Whole procedures is described in [5] The describable experiments were conducted on four young domestic pigs, each weighing approximately $20 \mathrm{~kg}$. The selection of the pig as an experimental animal was made in view of the high degree of functional similarity of the structure of pig skin to human skin. The animals were subjected to burns while under anaesthesia and analgesia. Each pig was usually subjected to 8 pairs of symmetrical wounds, covering approximately $1.5 \%$ of its total body surface area. As the final 30 burn wound with different depth was taken into account for further analysis. Assessment of burn wound depth by the clinical method and evaluation for prognosis of the ability of the wound to heal within three weeks following its occurrence was carried out 24 hours after the wound was inflicted. Photographic documentation was carried out on days: one, two, three, and 21 after the infliction of the burn wounds. IR camera synchronised with an excitation source (cooling by air/ $\mathrm{CO}_{2}$ - cryotherapy set). Changes of temperature are dependent on the internal structure of the tested object, vascularisation and blood flow intensity. Thermal responses to cooling pulse excitation are modelled as a two layer model which is describe by the two- exponentional equation ( $\mathrm{m}=2$ ):

$$
T(x, y, t)=T_{s}(x, y)+\sum_{j=1}^{m} \Delta T_{j}(x, y) \cdot\left(-e^{-\frac{t}{\tau_{j}(x, y)}}\right)
$$

During the nonlinear fitting to measured data procedure (Levenberg-Marquardt algorithm) five parameters $\left(T_{s}, \Delta T_{1}\right.$, $\left.\Delta T_{2}, \tau_{1}, \tau_{2}\right)$ are estimated. Time constants $\tau_{1}$ and $\tau_{2}$ may be of sufficient quality for medical diagnostics as classification parameters. Additional processing is required to improve the quality of the parametric images. First image to image 
registration should be performed, which eliminates image blurring and improves the signal to noise ratio of 1-D signals extracted from image sequences. The image registration algorithm needs a reference signs placed on the surface of the skin to normalise all the images in the sequence to the frame of reference - in practise to the first image. In clinical practice it is often situation that in the field of view there are visible signs which can be treated as markers for registration algorithm, e.g. border of bourn wound and healthy skin. It is also possible to use surface markers as a piece of bandage or other materials which should have different thermal properties than skin tissue.

However the parametric images of time constants are very useful in our opinion the better coefficient/estimator of burn depth is normalized time constant given by equation:

$$
\tau_{\text {norm }}=\frac{\tau-\bar{\tau}_{r e f}}{\tau+\bar{\tau}_{r e f}},
$$

where $\bar{\tau}_{\text {ref }}$ - mean $\tau$ for the reference healthy skin region.

Such definition eliminate the individual difference between patients and different states of the same patient e.g. fever or feverish what can change the blood flow intensity resulting in shorter time constant. When we normalize the discrimination parameter using the formula (2) using reference healthy region of the skin it is possible to establish universal for all population of patients threshold dividing the wound into two classes - wounds that will heal within 3 weeks and wounds that will not heal within 3 weeks. Such classification is very important from clinical point of view. Normalised time constant factor is equal to 0 for healthy region, is less then zero for superficial burn and greater then 0 for deeper wound.

3.

\section{Results}

The classification algorithm uses information about value and changes in time of the time constants factor. The classification criteria was establish according to in-vivo experimental results which are shown below. Figures 1 and 2 show the experimental analysis and results. Calculated time constant $\tau_{1}$ and $\tau_{2}$ respectively for the first and the second day following burn wound infliction for all wounds are presented. It can be observed that behaviour of parameters is quite different for the first and the second day. Such variability is due to healing and destroy processes which are present in a wound. Two photos of the burn wounds are presented in the figure below - the first one for the first day following the burn infliction and the second one after three weeks of treatment.
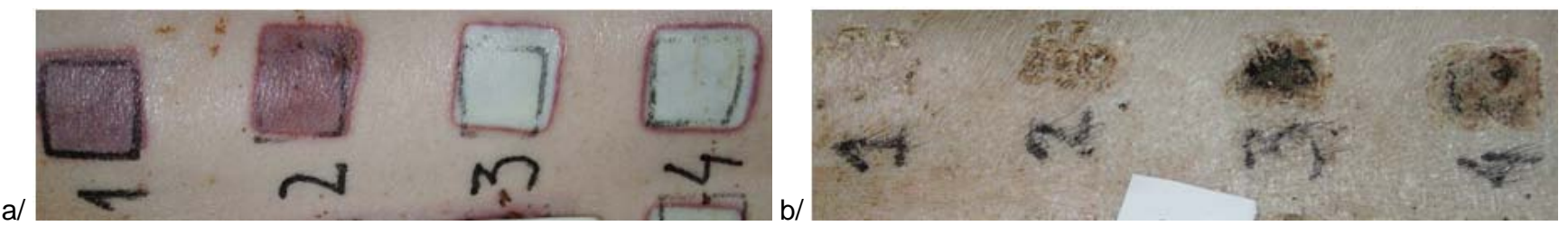

Fig. 1. The photographs of the burn wound: a/ the first day following burn infliction, $b / 21$ days after

Burns ROI (region of interest) "1" and "2" are the superficial burn while " 3 " and "4" are deeper one. So after 21 days of treatment burn "1" and "2" are healed but "3" and " 4 " are still covered by a scab. Clinical assessment suggest no healing of the wounds " 3 " and " 4 " within 3 weeks, the treatment decision should be surgery.

The results obtained and shown below were given as relative thickness, a percentage of the "dermis thickness at the measurement site" - dtms, and were subsequently used to calculate average values for all the elements undergoing assessment. The burn depth was assessed by determining the depth of damage to the following skin elements: hair follicle (epithelial cells), connective tissue collagen, nerves, smooth muscles (mesenhymal cells) and blood vessels. The depth was measured in millimetres starting from the epithelium basal layer.

For ADT the mean value of time constants for ROI numbered "1" to " 8 " were calculated and presented in fig. 2 and 3. We found that $\tau$ parameter had the lower value (was shorter) for wounds which self healed within 3 weeks than for the healthy skin, while $\tau$ had a higher value (was longer) for deeper wounds that failed to heal within this period. Due to destroy processes of the surface of a wound same ROI of superficial burns had a longer values of $\tau$ than the healthy skin. This cases should be excluded from further analysis. 

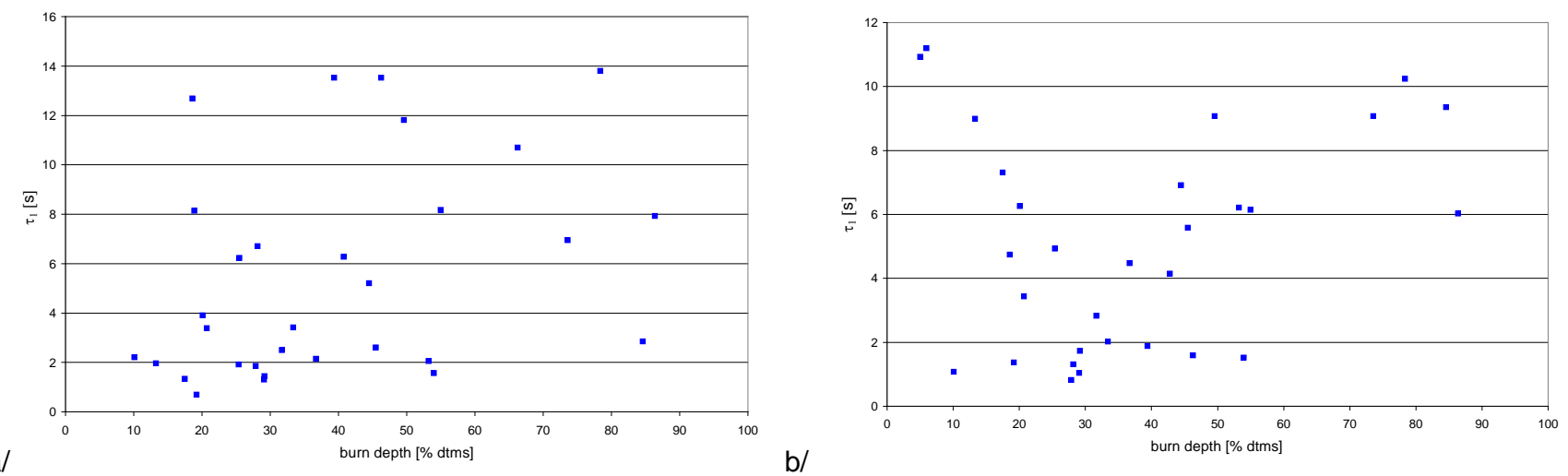

Fig. 2. Thermal time constants $\tau_{1}$ of burn wounds classified by histopathologic examination; a/ first day following accident, b/ second day following accident.

Analyzing thermal response the time constants are classified on 3 groups of different behaviour. The reference healthy skin secures all mechanisms characteristic for reaction to cooling by $\mathrm{CO}_{2}$ vapour. This is fast vasoconstriction to the cold stress (narrowing of the lumen of blood vessels), followed by vasodilatation - the opening of blood vessels - typical reaction after cooling, existing also in deeper layer of the tissue for longer periods of cooling. In effect the thermal response of the healthy skin is characterized by relatively long time constant for the short cooling and then due to increased vascularisation in deeper layers of the tissue, in response to longer cooling, it is keeping the time constant almost unchanged for increased periods of cooling. Burns which will healing within 3 weeks are lacking the effect of vasoconstriction and due to strong vascularisation at the superficial layer of the tissue the thermal response is fast. In contrast in the deep burn the perfusion of the tissue is poor and thermal response is much slower.

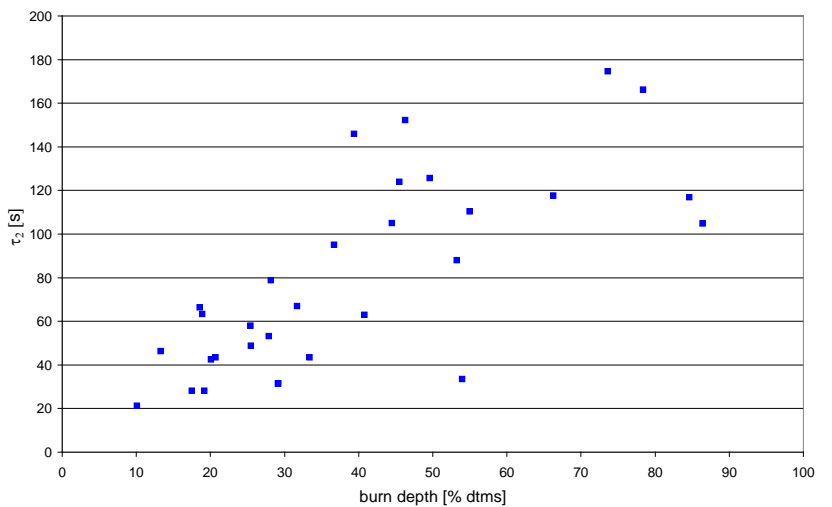

al

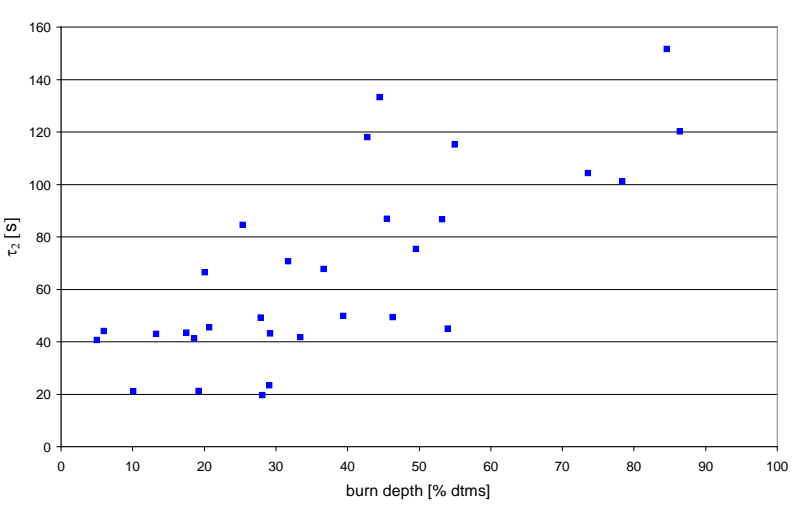

Fig. 3. Thermal time constants $\tau_{2}$ of burn wounds classified by histopathologic examination; a/ first day following accident, b/ second day following accident.

When the statistical analysis, clinical aspects of the problem and data from the literature are taken into account, the optimal time for the IRI investigations may be proposed as the second day after the burn.

Classifying the burn ROI to proper class of burn according to Jackson's criteria (I, IIA, IIB and III degree) and averaging obtained time constants it is difficult to calculate the discrimination thresholds onto four classes - figure 4 . The time constants for these ROI are to close to each other especially for first day following accident. Better situation is for the second day following burn accident.

For the clinical point of view dividing the burn wound onto two classes - spontaneously healed within 3 weeks and not healed within this period is very important. It can help the physician to take proper treatment procedure - to make surgery or not. Two class dividing is shown in figure 5 . The discrimination threshold was calculated for $\tau_{1}$ as equal 7 [s] and $\tau_{2}$ as equal 90 [s]. When these values were used for burn discrimination, the accuracy, sensitivity and specificity were all $100 \%$. However these thresholds can vary in quite big values according to localisation of the burn wound and individual characteristic of the patient. Skin thickness can vary from millimetres up to centimetres what impact the heat flow mechanisms and finally changed calculated time constant $\tau_{x}$. So, better way is to use the normalised time constant factor defined by equation 2 . The reference ROI of healthy skin should be chosen as close as it is possible to the burn wound. Then we are sure that skin has 
the same thickness as wound ROI and the excitation conditions was exactly the same. Calculated normalised time constant factors are presented in figures 7 and 8.

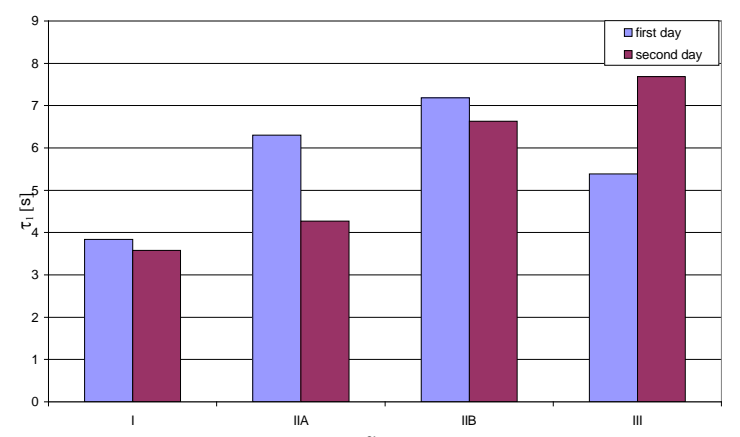

a/

classes

$\mathrm{b} /$

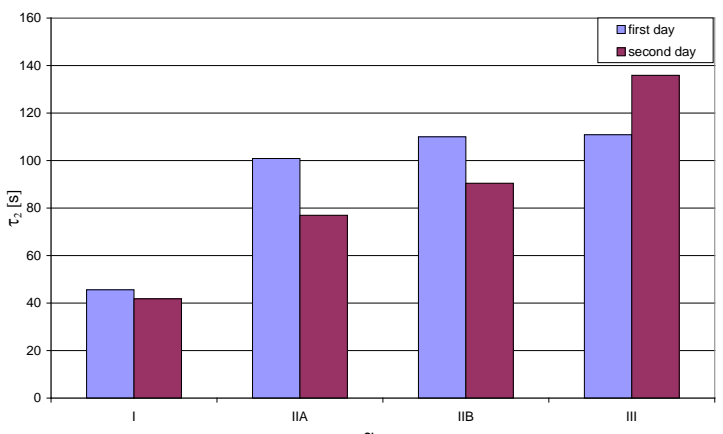

b/

Fig. 4. Thermal time constants of burn wounds classified by histopathologic examination as I, IIa, IIB, III degree - a/ $\tau_{1}, \mathrm{~b} / \tau_{2}$
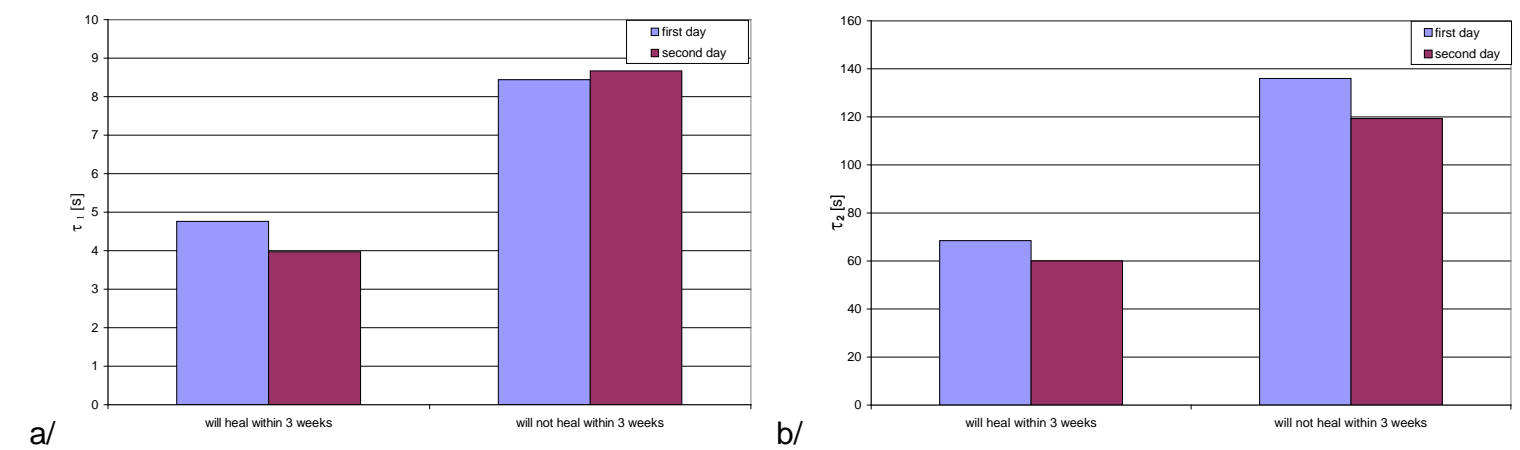

Fig. 5. Thermal time constants of burn wounds classified by histopathologic examination as "healed" and "non- healed" within 3 weeks - a/ $\tau_{1}$, b/ $\tau_{2}$.
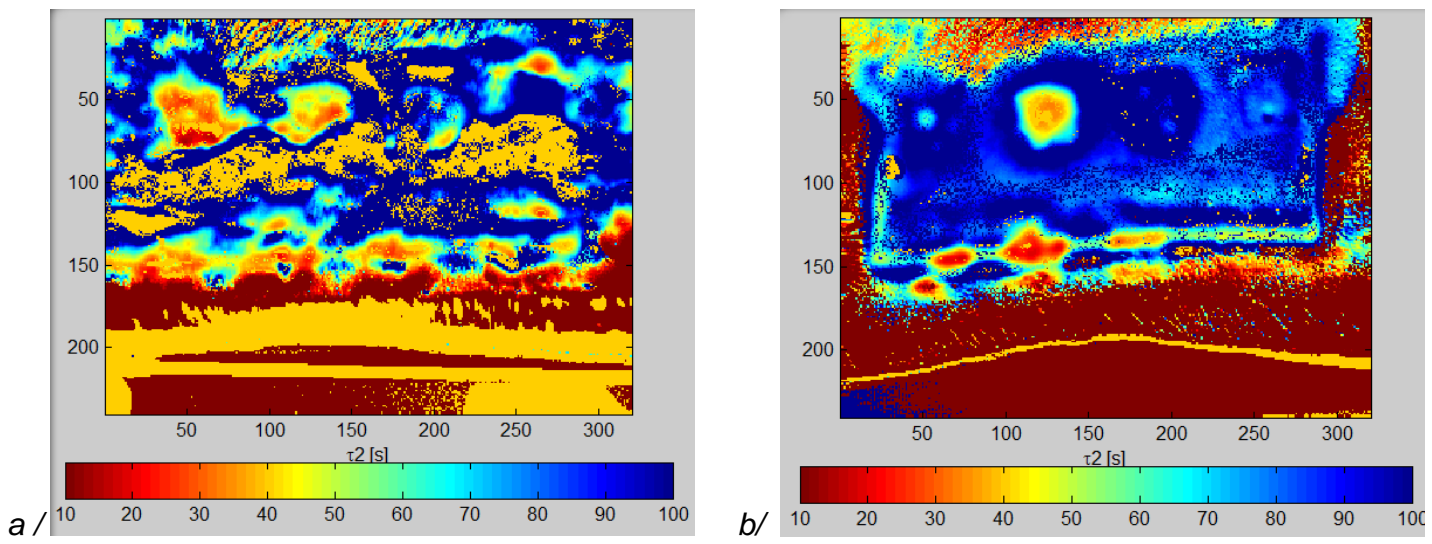

Fig. 6. Example of measurements - thermal time constants- two days observation - first day (left), and second day (right)

Figure 6 present the calculated parametric picture of time constant $\tau_{2}$ for whole field of view of thermal camera. The values of thermal constants are coded by colour according to colour scale. Longer time constants - deeper burn - are coded by blue and dark blue, when superficial burn are orange and red. Note, that all pixels with time constant longer than 90 [s] belongs to wound which not heal within 3 weeks and should had surgery. 

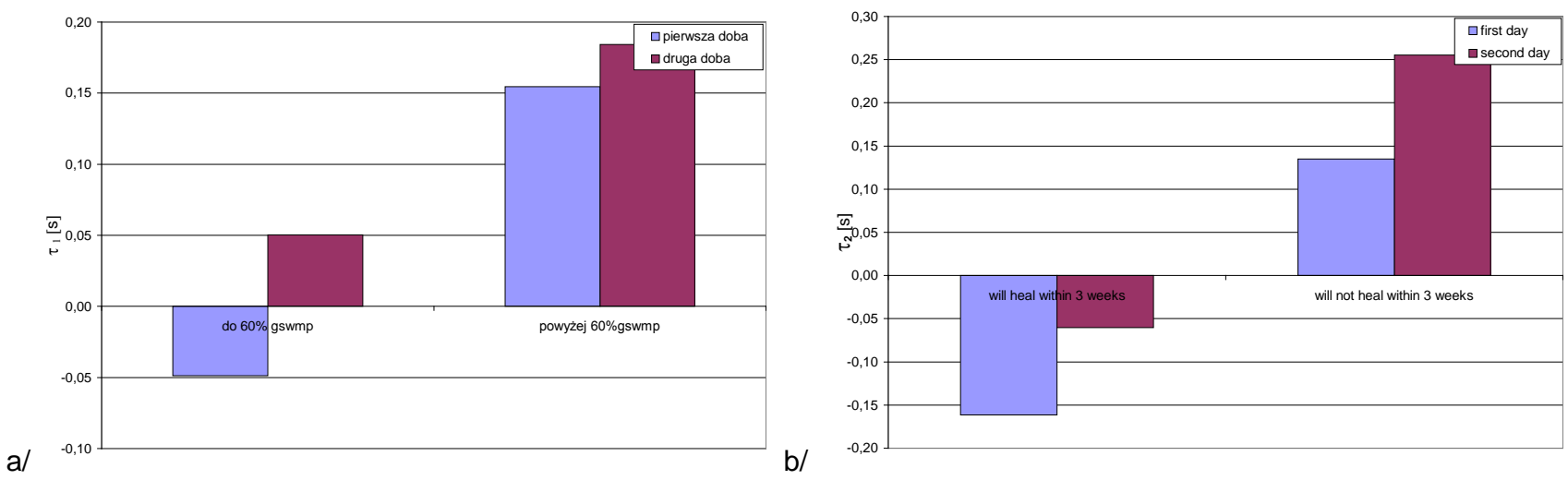

Fig. 7. Example of measurements - normalised thermal time constants factor - two days observation - first day (left), and second day (right)

Calculated parametric images of normalised time constant factor is shown in figure 9 for the first and second day following burn. The discrimination threshold was calculated for $\tau_{1}$ and $\tau_{2}$ as equal 0.06 . It is also proved by results presented in fig. 9 .
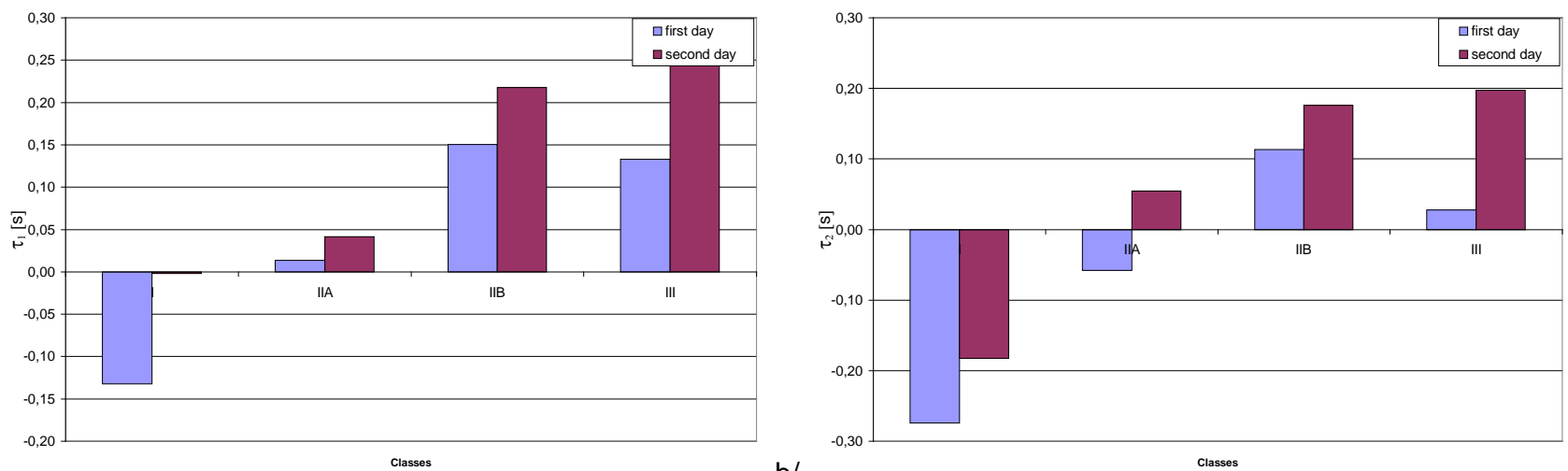

Fig. 8. Example of measurements - normalised thermal time constants factor - two days observation - first day (left), and second day (right)
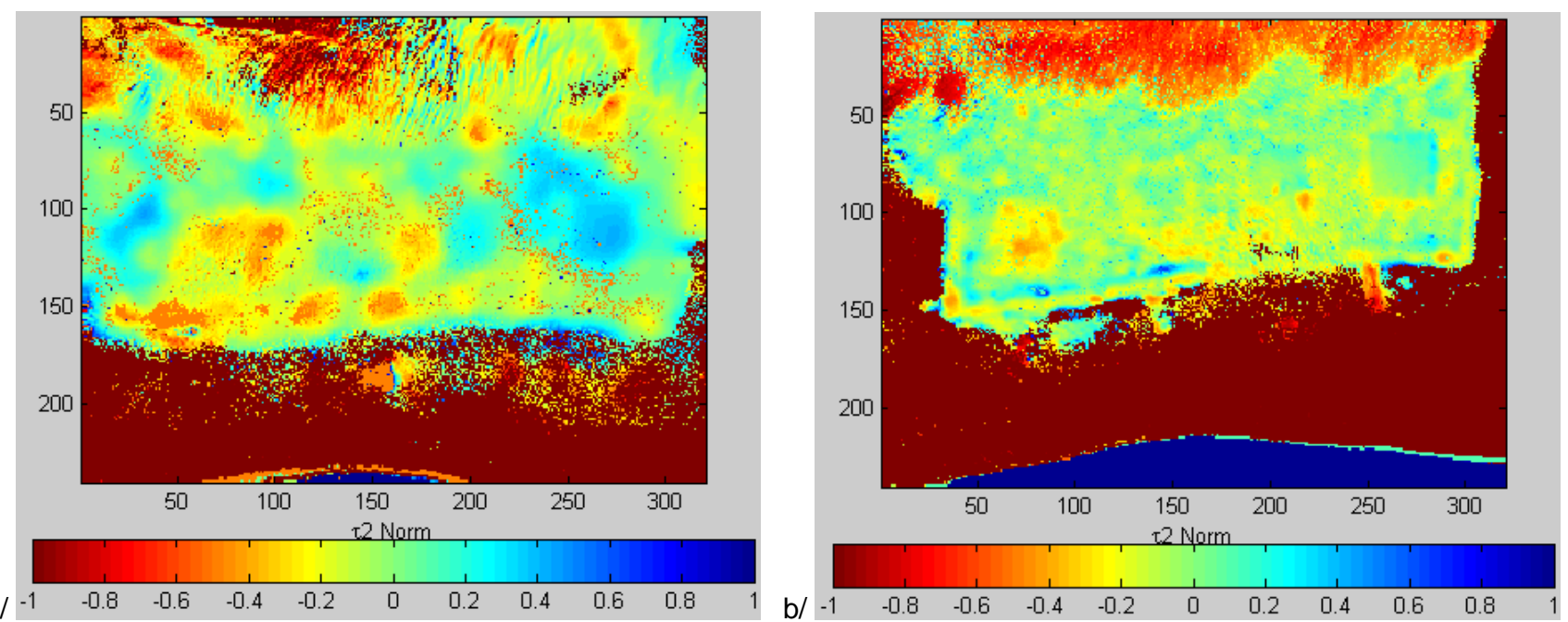

Fig. 9. Example of measurements - thermal time constants- two days observation - first day (left), and second day (right) 


\section{Clinical trials}

The ADT method has already been adopted for clinical use. As an example a burn wound on the abdomen and chest is shown in fig. 10. Investigation was done during next two days following accident.
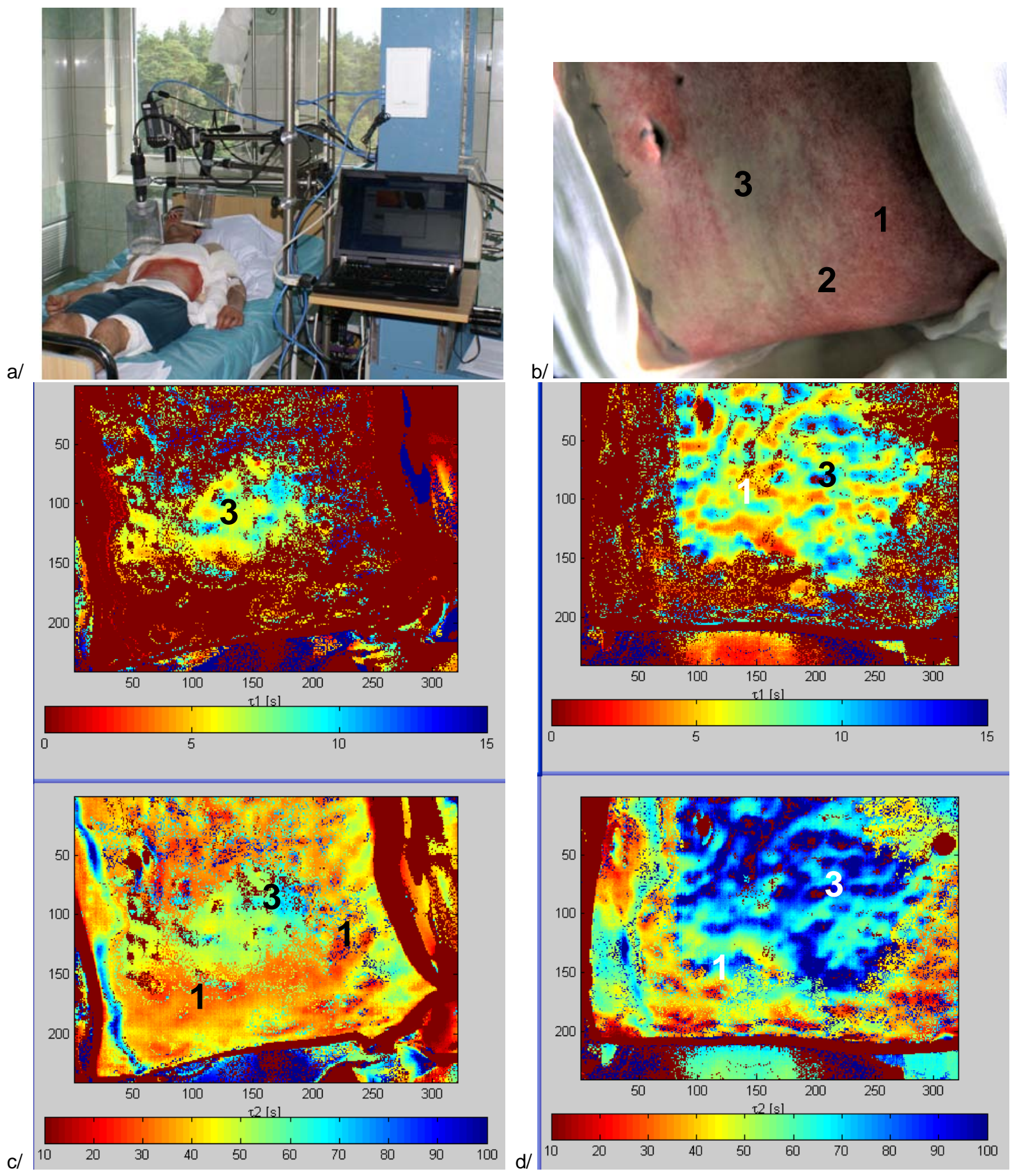

Fig. 10. Example of measurements; a/ photo of experimental setup, b/ photo of a wound; thermal time constants- two days observation - first day - c/, and second day - d/ 
In figure 10 b markers "1", "2" and " 3 " denote the clinical prognosis of burn depth - "1" - superficial burn, will heal within 3 weeks, "3" - deep burn will not heal within 3 weeks and "2" - hard to say, it need further observation. By analogy with markers in figure 10b, markers "1" and " 3 " in figures 10 /c and /d means respectively: wound which will heal within 3 weeks and wound which not heal within 3 weeks. One can observe that results obtained for the second day following accident shows the wider region of deep burn which will not heal within 3 weeks. It is due to destroy processes ongoing in wound. No histopathologic data was collected in this case, so only ex-post observation proved the thermal imaging conclusions about the state of burn.

\section{Discussion}

The depth of experimental burn wounds has been evaluated by means of 4 diagnostic methods: clinical observation, traditional thermography, ADT and histopathology (as the reference). Discrimination of regions of limited vascularisation or necrosis but also regions of increased metabolism and tissues of affected properties are possible. The information taken from static thermography and active dynamic thermography (ADT) allows to make direct inspection of the state of a tested organ, e.g. the heart or a skin burn, giving quantitative evidence of applied surgical procedures.

The quantitative synthetic parameter of ADT, the time constant T measured in [s], supplies information about the basic physical thermal parameters of the tissue. Possessing all the advantages of Static Thermography (ST), ADT is much less subject to the limitations of ST. When all this is taken into consideration it may be claimed that ADT meets to the highest degree the requirements of a modern diagnostic method which will evaluate burn depth and, in consequence, prove useful in the proper choice of treatment. It is concluded that IRI in its complex modern scope is of great use in the assessment of burn wound depth, especially in the face of the difficult and clinically crucial problem of making the appropriate choice of treatment. The optimal time for the investigation is the second day after the burn.

5.

\section{Conclusion}

ADT examination is simple, non-contact and short (up to few minutes). The instrumentation to be applied is based on IR-cameras already available in hospitals and as external excitation source the cryotherapy unit. The procedure is not as sensitive to external conditions as static thermography. The normalised time constant factor is quantitative, enabling burns to be objectively classified and proper treated. The results of the ADT and histopathological evaluations are fully in agreement what is not always true for static thermography.

\section{Acknowledgment}

Author has greatly appreciated the participation of prof. A. Renkielska and Dr J. Grudziński from the Department of Plastic Surgery of Gdansk Medical University, Dr W. Stojek from the Department of Animal Physiology of Gdansk University and colleagues from the Department of Biomedical Engineering Gdansk University of Technology.

\section{REFERENCES}

[1] Rumiński J., Kaczmarek M., Renkielska A., Nowakowski A., Thermal parametric imaging in the evaluation of skin burn depth, IEEE Transaction on Biomedical Engineering, Vol. 54, 2, pp. 303-312, 2007.

[2] Chatterjee J.S., A Critical Evaluation of the Clinimetrics of Laser Doppler as a Method of Burn Assessment in Clinical Practice, Journal of Burn Care \& Research, 27, p. 123 - 130, 2006.

[3] Green H. A., Bua D.,Anderson R. R., Nishioka N. S.:Burn depth estimation using indocyanine green fluorescence. Arch. Dermatol., 128, p. 43-49, 1992.

[4] Singer A.J., Berruti L., Thode HC J.R., McClain S.A., Standardised burn model using a multiparametric histologic analysis of burn depth. Academic Emergency Medicine, vol. 7:1, pp. 1-6, 2000.

[5] Renkielska A., Nowakowski A., Kaczmarek M., Dobke M., Grudziński J., Karmoliński A., Stojek W., Static thermography revisited - An adjunct method for determining the depth of the burn injury, Burns, v. 31, (6), 768-775, 2005. 